\title{
SOPHE Computation on Alanine Single Crystal EPR Spectra
}

\author{
Frida U. Ermawati \\ Physics Dept. Surabaya State University \\ Email: frida_dua@yahoo.com
}

\begin{abstract}
Simulation of X-band first-derivative continuous-wave electron paramagnetic resonance $(C W$-EPR) experimental spectra of $\gamma$-irradiated $\ell-\alpha$-alanine single crystal has been carried out. The aim was to confirm the existence of radical induced by irradiation in the system. A SOPHE computer simulation software suite (v. 1.1.3) was utilized for that purpose. Six different $X$-band spectra measured from a number of defined crystal orientations to the external magnetic field B were recorded and simulated. The "axial" spectra (refer to the simulated spectra when the external B field was parallel to the crystal axes and that was measured at room temperature) confirm the simultaneous presence of the $S A R, R 2$ and $R 3$ radicals with the appropriate proportions. The "planar" spectra (refer to the simulated spectra when the B field was on the crystal planes), however, do not exactly fit to the experimental spectra. Together with the simulation outcomes, the problems that appear during the work, as well as the solutions proposed to overcome those problems are discussed.
\end{abstract}

Keywords: $\ell-\alpha$-alanine, SAR, Computer simulation, SOPHE, Xepr. 


\section{Introduction}

It is quite clear that nowadays computer simulation has become increasingly important in many branches of science. In the area of electron paramagnetic resonance (EPR), computer simulation of an EPR spectrum is a normal procedure in data analysis. There are two different attitudes to the role played by computer simulation in that area, namely: on the one hand, the purist might wish to examine a particular spectrum in very great detail. On the other hand, a large number of spectra as function of the various input parameters could be generated by the availability of a computer program. For example, spectral simulations of highly anisotropic systems require improved and efficient methods of computer simulation whereby quantification of unknown spectra can be achieved. Therefore, computer simulation often provides the experimenter with insight into what parameters are important and what modeling is relevant to the problems.

The work reported in this paper is intended to establish a confirmation of simultaneous presence of radicals in $\ell-\alpha$ alanine single crystal, an EPR/radiation dosimetry material, by taking agreement between the simulated and the experimental CW-EPR spectra. SOPHE computer simulation software suite version 1.1.3 developed in the Center for Magnetic Resonance and the Department of Mathematics, The University of Queensland $^{1)}$ was utilized throughout this work.

\section{Theoretical background and} experimental results: A Brief Review Symmetry related paramagnetic sites in $\ell$ $\alpha$-alanine

$\ell$ - $\alpha$-Alanine (see Fig.1a) is an orthorhombic crystal whose space group is $P 22_{1} 2_{1}$. It contains four molecules, hence four paramagnetic sites (see Fig.2) in a unit cell whose positions can be generated by applying two-fold screw axis symmetry to the original position. As shown, those four site positions are namely: (I) - the original position, (I to IV) - $(2 \pi / 2)$ rotation of the original position, each about $\langle\mathrm{b}\rangle$, $\langle a\rangle$ and $\langle\mathrm{c}\rangle$ crystal axes followed by translation along those axes. If coordinate of the first-site (or the original position) is $(x, y, z)$, coordinates of the second-, the third- and the fourth-sites are $(\underline{x}, \underline{y}, z),(\underline{x}, y, \underline{z})$ and $(x, y, z)$, respectively, where underlines indicate the coordinates are negative with respect to the origin. a)<smiles>CC(N)C(=O)[O-]</smiles>

b)<smiles>CC(C)C(=O)O</smiles>

c)<smiles>C[C](N)C(=O)[O-]</smiles>

d)<smiles>CC(N)C([O])C=CNC(=O)C(O)C=CNC(Cl)C(=O)O</smiles>

Fig. 1: The proposed structure of: a) $\ell$ - $\alpha$-alanine, b) stable alanine radical (SAR) c) R2 radical, and d) two conformations of R3 radical ${ }^{2-3)}$

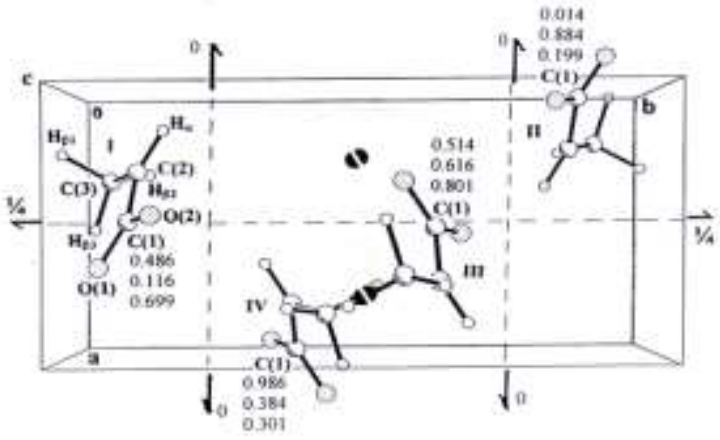

Fig. 2: The four (I-IV) paramagnetic sites of $\ell-\alpha$ alanine in a unit cell, viewed along $\langle c\rangle$ crystal axis. Those positions were generated by the author of this paper from the SAR coordinates provided by Kuroda and Miyagawa, $(1982)^{3)}$. Fractions of the four (C1) atomic positions with respect to the origin $O$ were also labeled. The dash lines and $\mathbf{1 0}$ symbol show the two-fold screw axis symmetry to generate the four sites as described in the text. 


\section{Experimental results}

In this work, X-band CW-EPR resonance spectra of the first-derivative of the absorption lines of 28 kGray, $\gamma$ irradiated of homegrown $\ell$ - $\alpha$-alanine single crystal were measured at room temperature (RT) by using a Varian E-12 EPR spectrometer. Fig.3-4 show six different experimental spectra when the external dc magnetic (B) field was set parallel to crystal axes as well as on the crystal planes. A goniometer with two degrees of freedom was previously set in the cavity of
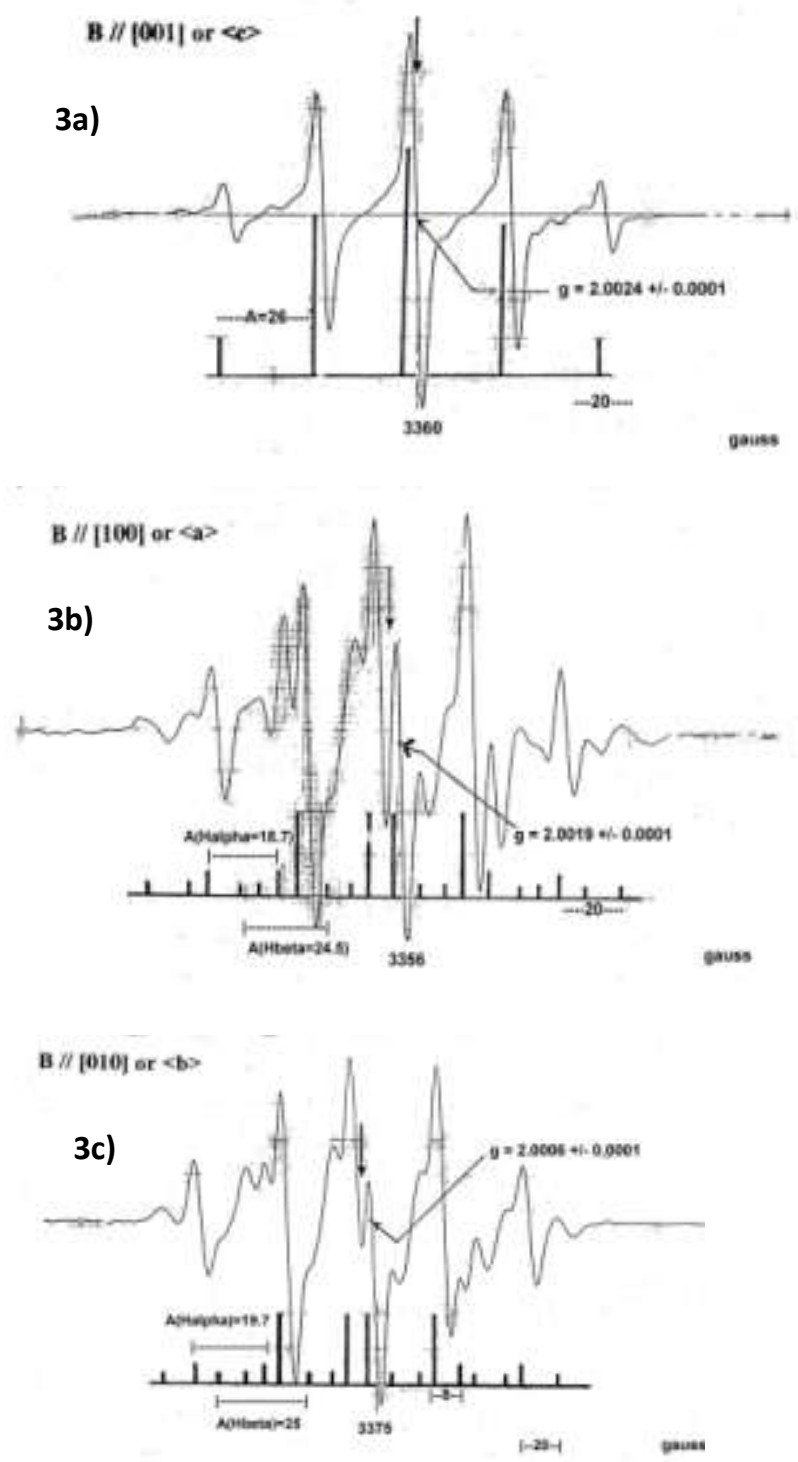

the spectrometer and utilized in this work to fix the crystal such that the desired crystal orientations with respect to the external B field can be achieved. Angular coordinates of the crystal were measured both from polar $(\theta)$ and azimuthal $(\phi)$ scales on the goniometer head. The protocol was: $1 \mathrm{~mW}$ of microwave power and its frequency was $9.45 \mathrm{GHz}$, the external B field was centered to 3360 gauss and the swept through 200 gauss. Modulation amplitude and its frequency were 0.4 gauss and $100 \mathrm{kHz}$, respectively.

Fig. 3a): First-derivative CW-EPR spectrum of $\gamma$-irradiated $l$ $\alpha$-alanine single crystal measured at RT when the external $B$ field was parallel to $\langle$ c $>$ crystal axis. A quintet pattern with the relative intensity ratio close to $1: 4: 6: 4: 1$ was featuring the spectrum. To the first order of approximation (see the stick diagram under the spectrum) this was due to four equivalent proton couplings of 26 gauss, $\alpha$-proton and three protons of rotating methyl group. The g-factor to each of which the spectrum was centered is also labeled. The arrow pointing down indicates the position of the g-factor for the DPPH sample, 2.0036 .

Fig. 3b-c): Similar spectra as in Fig. 3a, but it was recorded when the external $B$ field was parallel to: $\langle a\rangle$ (see Fig. $3 b$ ) and $\langle b\rangle$ (Fig.3c). A quartet of line pairs with the relative intensity ratio approximately to $1: 1: 3: 3: 3: 3: 1: 1$ dominates the pattern. The stick diagrams under the spectra calculated to the second-order of approximation clarify the results. The symbols used are the same as in Fig. 3a. The splitting due to $H_{\alpha}$ and $H_{\beta}$ are also shown. 


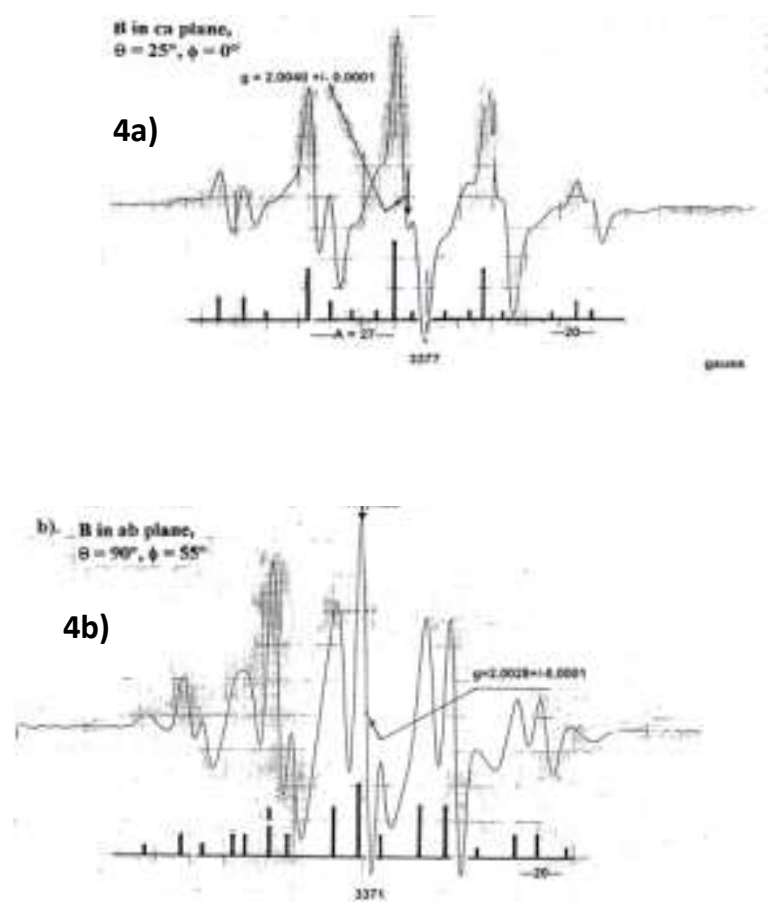

Transformation of the $g$ - and the hyperfine coupling tensors of the four sites in $\ell-\alpha$ alanine crystals due to $S A R, R 2$ and $R 3$ radicals

Free radical formation in $\mathrm{X}$-irradiated at room temperature of $\ell$ - $\alpha$-alanine crystals has been studied extensively by $\mathrm{X}$ - and $\mathrm{K}$ bands CW-EPR ${ }^{2-5)}$, Pulsed EPR ${ }^{4)}$, Electron nuclear double resonance (ENDOR) and ENDOR induced EPR (EIE) $)^{2,3,5)}$ in the temperature range $220-295 \mathrm{~K}$ and $77 \mathrm{~K}$. In that works ${ }^{2-5)}$, the $\mathrm{g}$ - and the nine-hyperfine coupling tensors were obtained and assigned to three different radicals, that is, the R1 (stable alanine radical, SAR), R2 and $\mathrm{R} 3$ radical, a minority species. Tables 1-3 recapitulate matrices of the $\mathrm{g}$ - and the hyperfine coupling tensors, each for SAR, R2 and two conformations of minor species R3 radical. The matrices were calculated by the author of this paper based on the ENDOR data given by the Ref. No. 2. Second rank transformation law ${ }^{6}$ of

$$
\mathrm{T}^{\prime}{ }_{\mathrm{ij}}=\mathrm{a}_{\mathrm{ik}} \mathrm{a}_{\mathrm{j} 1} \mathrm{~T}_{\mathrm{kl}}
$$

Fig. 4a-c): Similar spectra as in Fig. 3a-c, but they were recorded when the external $B$ field was on: Left, a) c-a plane $\left(\theta=25^{\circ}, \phi=0^{\circ}\right)$. Below, b) a-b plane $\left(\theta=90^{\circ}, \phi=55^{\circ}\right)$ and c) b-c plane $\left(\theta=55^{\circ}, \phi=90^{\circ}\right)$.

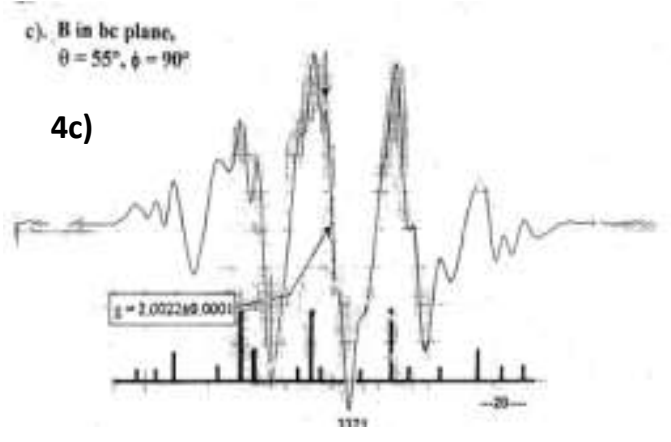

was followed to transform the g- and the hyperfine coupling tensors of the system. In Eq. (1) $\mathrm{T}_{\mathrm{ij}}$ and $\mathrm{T}_{\mathrm{kl}}$ are the 'old' and 'new' second rank tensors whose elements have been transformed to the crystal axes; $\mathrm{a}_{\mathrm{ik}}$ and $\mathrm{a}_{\mathrm{jl}}$ are matrix elements of the direction cosines; $\mathrm{k}$ and $\mathrm{l}$ are dummy suffices while $\mathrm{i}$ and $\mathrm{j}$ are free suffices. Tensor transformation from individual principal axes to crystallographic axes is prerequisite in simulation work presented in this paper. The g- and the hyperfine coupling tensor elements in Tables 1-3 were given in $\mathrm{MHz}$ unit. The uncertainty was given at the $95 \%$ confidence level in the last digit(s) of the quoted values.

\section{Stable alanine radical (SAR)}

The SAR (Fig. 1b) was assigned from three interactions based on $\mathrm{X}$-band ENDOR and EPR measurements at $295 \mathrm{~K}$ by locking the external magnetic field in various positions which was parallel with $<\mathrm{a}\rangle$ and $\langle\mathrm{c}\rangle$ crystal axes. 
Table-1: Matrices of the transformed g-and the hyperfine coupling tensors due to SAR in $\ell$ - $\alpha$-alanine generated for the four sites of the system based on the ENDOR data given in Ref. No. 2.

\begin{tabular}{|c|c|c|c|c|c|c|c|c|c|c|c|}
\hline \multicolumn{12}{|c|}{ The g-tensor (in $\mathrm{MHz}$ ) } \\
\hline \multicolumn{3}{|l|}{ SITE-1: } & \multicolumn{3}{|l|}{ SITE-2: } & \multicolumn{3}{|c|}{ SITE-3: } & \multicolumn{3}{|l|}{ SITE-4: } \\
\hline 2.0034 & 0.0012 & -0.0012 & 2.0034 & 0.0012 & 0.0012 & 2.0034 & -0.0012 & -0.0012 & 2.0034 & -0.0012 & 0.0012 \\
\hline 0.0012 & 2.0044 & 0.0010 & 0.0012 & 2.0044 & -0.0010 & -0.0012 & 2.0044 & -0.0010 & -0.0012 & 2.0044 & 0.0010 \\
\hline-0.0012 & 0.0010 & 2.0024 & 0.0012 & -0.0010 & 2.0024 & -0.0012 & -0.0010 & 2.0024 & 0.0012 & 0.0010 & 2.0024 \\
\hline \multicolumn{12}{|c|}{ The $\mathrm{H}_{\alpha}$-tensors (in $\mathrm{MHz}$ ) } \\
\hline \multicolumn{3}{|l|}{ SITE-1: } & \multicolumn{3}{|l|}{ SITE-2: } & \multicolumn{3}{|c|}{ SITE-3: } & \multicolumn{3}{|l|}{ SITE-4: } \\
\hline$-43.5(2)$ & $-17.2(2)$ & $-17.4(1)$ & $-43.5(2)$ & $-17.2(2)$ & $17.4(1)$ & $-43.5(2)$ & $17.2(2)$ & $-17.4(1)$ & $-43.5(2)$ & $17.2(2)$ & $17.4(1)$ \\
\hline$-17.2(2)$ & $-51.9(2)$ & $-8.8(2)$ & $-17.2(2)$ & $-51.9(2)$ & $-8.8(2)$ & $17.2(2)$ & $-51.9(2)$ & $8.8(2)$ & $17.2(2)$ & $-51.9(2)$ & $-8.8(2)$ \\
\hline$-17.4(1)$ & $-8.8(2)$ & $-72.8(1)$ & $17.4(1)$ & $-8.8(2)$ & $-72.8(1)$ & $-17.4(1)$ & $8.8(2)$ & $-72.8(1)$ & $17.4(1)$ & $-8.8(2)$ & $-72.8(1)$ \\
\hline \multicolumn{12}{|c|}{ The $\mathrm{H}_{\beta}$-tensors (in $\mathrm{MHz}$ ) } \\
\hline \multicolumn{3}{|l|}{ SITE-1: } & \multicolumn{3}{|l|}{ SITE-2: } & \multicolumn{3}{|c|}{ SITE-3: } & \multicolumn{3}{|l|}{ SITE-4: } \\
\hline $67.7(1)$ & $-0.6(4)$ & $-1.0(4)$ & $67.7(1)$ & $-0.6(4)$ & $1.0(4)$ & $67.7(1)$ & $0.6(4)$ & $-1.0(4)$ & $67.7(1)$ & $0.6(4)$ & $1.0(4)$ \\
\hline$-0.6(4)$ & $69.9(20$ & $3.4(3)$ & $-0.6(4)$ & $69.9(2)$ & $-3.4(3)$ & $0.6(4)$ & 69.9(2) & $-3.4(3)$ & $0.6(4)$ & 69.9(2) & $3.4(3)$ \\
\hline$-1.0(4)$ & $3.4(3)$ & $71.8(2)$ & $1.0(4)$ & $-3.4(3)$ & $71.8(2)$ & $-1.0(4)$ & $-3.4(3)$ & $71.8(2)$ & $1.0(4)$ & $3.4(3)$ & $71.8(2)$ \\
\hline \multicolumn{12}{|c|}{ The $\mathrm{H}_{\text {dip }}$ tensors (in $\mathrm{MHz}$ ) } \\
\hline \multicolumn{3}{|l|}{ SITE-1: } & \multicolumn{3}{|l|}{ SITE-2: } & \multicolumn{3}{|l|}{ SITE-3: } & \multicolumn{3}{|l|}{ SITE-4: } \\
\hline$-1.8(2)$ & $1.1(3)$ & $-0.9(3)$ & $-1.8(2)$ & $1.1(3)$ & $0.9(3)$ & $-1.8(2)$ & $-1.1(3)$ & $-0.9(3)$ & $-1.8(2)$ & $-1.1(3)$ & $0.9(3)$ \\
\hline $1.1(3)$ & $2.4(2)$ & $-3.3(2)$ & $1.1(3)$ & $2.4(2)$ & $3.3(2)$ & $-1.1(3)$ & $2.4(2)$ & $3.3(2)$ & $-1.1(3)$ & $2.4(2)$ & $-3.3(2)$ \\
\hline$-0.9(3)$ & $-3.3(2)$ & $-0.2(2)$ & $0.9(3)$ & $3.3(2)$ & $-0.2(2)$ & $-0.9(3)$ & $3.3(2)$ & $-0.2(2)$ & $0.9(3)$ & $-3.3(2)$ & $-0.2(2)$ \\
\hline
\end{tabular}

\section{$R 2$ radical: The $H$-abstraction radical}

The second R2 radical (Fig. 1c) was formed by a net $\mathrm{H}$-abstraction from the $\mathrm{C}_{\alpha^{-}}$ position in $\ell-\alpha$-alanine. The existence of the $\mathrm{R} 2$ radical was assigned from the resonance features outside of the resonance due to SAR in $\mathrm{X}$ - and $\gamma$-irradiated $\ell$ - $\alpha$ alanine single crystal spectra. These features remain weak in all EPR spectra. Table-2 lists the transformed hyperfine coupling tensors for $\mathrm{R} 2$ radical.

Table-2: The transformed hyperfine coupling tensors of R2 radical, calculated from ENDOR data in Ref. No. 2.

\begin{tabular}{|c|c|c|c|c|c|c|c|c|c|c|c|}
\hline \multicolumn{12}{|c|}{ The $\mathrm{H}_{\beta}$-tensors (in $\mathrm{MHz}$ ) } \\
\hline \multicolumn{3}{|l|}{ SITE-1: } & \multicolumn{3}{|l|}{ SITE-2: } & \multicolumn{3}{|c|}{ SITE-3: } & \multicolumn{3}{|l|}{ SITE-4: } \\
\hline $74.9(2)$ & $1.9(3)$ & $2.1(3)$ & $74.9(2)$ & $1.9(3)$ & $-2.1(3)$ & $74.9(2)$ & $-1.9(3)$ & $2.1(3)$ & $74.9(2)$ & $-1.9(3)$ & $-2.1(3)$ \\
\hline $1.9(3)$ & $68.5(1)$ & $0.4(2)$ & $1.9(3)$ & $68.5(1)$ & $-0.4(2)$ & $-1.9(3)$ & $68.5(1)$ & $-0.4(2)$ & $-1.9(3)$ & $68.5(1)$ & $0.4(2)$ \\
\hline $2.1(3)$ & $0.4(2)$ & $68.8(1)$ & $-2.1(3)$ & $-0.4(2)$ & $68.8(1)$ & $2.1(3)$ & $-0.4(2)$ & $68.8(1)$ & $-2.1(3)$ & $0.4(2)$ & $68.8(1)$ \\
\hline \multicolumn{12}{|c|}{ The $\mathrm{H}_{\beta(1) \mathrm{N}}$-tensors (in $\mathrm{MHz}$ ) } \\
\hline \multicolumn{3}{|l|}{ SITE-1: } & \multicolumn{3}{|l|}{ SITE-2: } & \multicolumn{3}{|c|}{ SITE-3: } & \multicolumn{3}{|l|}{ SITE-4: } \\
\hline $87.1(3)$ & $-5.1(4)$ & $-6.2(4)$ & $87.1(3)$ & $-5.1(4)$ & $6.2(4)$ & $87.1(3)$ & $5.1(4)$ & $-6.2(4)$ & $87.1(3)$ & $5.1(4)$ & $6.2(4)$ \\
\hline$-5.1(4)$ & $86.4(3)$ & $2.4(3)$ & $-5.1(4)$ & $86.4(3)$ & $-2.4(3)$ & $5.1(4)$ & $86.4(3)$ & $-2.4(3)$ & $5.1(4)$ & $86.4(3)$ & $2.4(3)$ \\
\hline$-6.2(4)$ & $2.4(3)$ & $85.1(2)$ & $6.2(4)$ & $-2.4(3)$ & $85.1(2)$ & $-6.2(4)$ & $-2.4(3)$ & $85.1(2)$ & $6.2(4)$ & $2.4(3)$ & $85.1(2)$ \\
\hline \multicolumn{12}{|c|}{ The $\mathrm{H}_{\beta(2) \mathrm{N}}$-tensors (in $\mathrm{MHz}$ ) } \\
\hline \multicolumn{3}{|l|}{ SITE-1: } & \multicolumn{3}{|l|}{ SITE-2: } & \multicolumn{3}{|l|}{ SITE-3: } & \multicolumn{3}{|l|}{ SITE-4: } \\
\hline $37.7(2)$ & $4.2(3)$ & $-4.8(3)$ & $37.7(2)$ & $4.1(3)$ & $4.8(3)$ & $37.7(2)$ & $-4.1(3)$ & $-4.8(3)$ & $37.7(2)$ & $-4.1(3)$ & $4.8(3)$ \\
\hline $4.1(3)$ & $25.3(2)$ & $-1.3(3)$ & $4.1(3)$ & $25.3(2)$ & $1.3(3)$ & $-4.1(3)$ & $25.3(2)$ & $1.3(3)$ & $-4.1(3)$ & $25.3(2)$ & $-1.3(3)$ \\
\hline$-4.8(3)$ & $-1.3(3)$ & $27.3(3)$ & $4.8(3)$ & $1.3(3)$ & $27.3(3)$ & $-4.8(3)$ & $1.3(3)$ & $27.3(3)$ & $4.8(3)$ & $-1.3(3)$ & $27.3(3)$ \\
\hline \multicolumn{12}{|c|}{ The $\mathrm{H}_{3(3) \mathrm{N}}$-tensors (in $\mathrm{MHz}$ ) } \\
\hline \multicolumn{3}{|l|}{ SITE-1: } & \multicolumn{3}{|l|}{ SITE-2: } & \multicolumn{3}{|l|}{ SITE-3: } & \multicolumn{3}{|l|}{ SITE-4: } \\
\hline $5.9(3)$ & $-0.1(2)$ & $-2.8(2)$ & $5.9(3)$ & $-0.1(2)$ & $2.8(2)$ & $5.9(3)$ & $0.1(2)$ & $-2.8(2)$ & $5.9(3)$ & $0.1(2)$ & $2.8(2)$ \\
\hline$-0.1(2)$ & $5.4(2)$ & $0.4(3)$ & $-0.1(2)$ & $5.4(2)$ & $-0.4(3)$ & $0.1(2)$ & $5.4(2)$ & $-0.4(3)$ & $0.1(2)$ & $5.4(2)$ & $0.4(3)$ \\
\hline$-2.8(2)$ & $0.4(3)$ & $19.3(2)$ & $2.8(2)$ & $-0.4(3)$ & $19.3(2)$ & $-2.8(2)$ & $-0.4(3)$ & $19.3(2)$ & $2.8(2)$ & $0.4(3)$ & $19.3(2)$ \\
\hline
\end{tabular}

\section{$R 3$ radical: A minority species}

The minor species of R3 radical (Figure 1d) resides together with the SAR. The existence is featuring the resonance lines due to SAR. Table-3 lists the transformed hyperfine coupling tensors for the two conformations of the R3 radical, based on the data in the Ref. No. 2. The tensors are characteristic of coupling between the unpaired electron and a freely rotating methyl group. 
Table-3: The transformed hyperfine coupling tensors of two conformations of R3 radical, based on the ENDOR data in Ref. No.2.

\begin{tabular}{|c|c|c|c|c|c|c|c|c|c|c|c|}
\hline \multicolumn{12}{|c|}{ The $\mathrm{H}_{\beta}$-tensors (in $\mathrm{MHz}$ ) } \\
\hline \multicolumn{3}{|l|}{ SITE-1: } & \multicolumn{3}{|l|}{ SITE-2: } & \multicolumn{3}{|c|}{ SITE-3: } & \multicolumn{3}{|l|}{ SITE-4: } \\
\hline $43.8(2)$ & $1.9(2)$ & $1.1(3)$ & $43.8(2)$ & $1.9(2)$ & $-1.1(3)$ & $43.8(2)$ & $-1.9(2)$ & $1.1(3)$ & $43.8(2)$ & $-1.9(2)$ & $-1.1(3)$ \\
\hline $1.9(2)$ & $37.6(2)$ & $0.0(3)$ & $1.9(2)$ & $37.6(2)$ & $-0.0(3)$ & $-1.9(2)$ & $37.6(2)$ & $-0.0(3)$ & $-1.9(2)$ & $37.6(2)$ & $0.0(3)$ \\
\hline $1.1(3)$ & $0.0(3)$ & $37.0(2)$ & $-1.1(3)$ & $-0.0(3)$ & $37.0(2)$ & $1.1(3)$ & $-0.0(3)$ & $37.0(2)$ & $-1.1(3)$ & $0.0(3)$ & $37.0(2)$ \\
\hline \multicolumn{12}{|c|}{ The $\mathrm{H}_{\beta}$-tensors (in $\mathrm{MHz}$ ) } \\
\hline \multicolumn{3}{|l|}{ SITE-1: } & \multicolumn{3}{|l|}{ SITE-2: } & \multicolumn{3}{|l|}{ SITE-3: } & \multicolumn{3}{|l|}{ SITE-4: } \\
\hline $35.9(2)$ & $2.5(3)$ & $1.6(2)$ & $35.9(2)$ & $2.5(3)$ & $-1.6(2)$ & $35.9(2)$ & $-2.5(3)$ & $1.6(2)$ & $35.9(2)$ & $-2.5(3)$ & $-1.6(2)$ \\
\hline $2.5(3)$ & $32.0(3)$ & $0.8(2)$ & $2.5(3)$ & $32.0(3)$ & $-0.8(2)$ & $-2.5(3)$ & $32.0(3)$ & $-0.8(2)$ & $-2.5(3)$ & $32.0(3)$ & $0.8(2)$ \\
\hline $1.6(2)$ & $0.8(2)$ & $31.3(3)$ & $-1.6(2)$ & $-0.8(2)$ & $31.3(3)$ & $1.6(2)$ & $-0.8(2)$ & $31.3(3)$ & $-1.6(2)$ & $0.8(2)$ & $31.3(3)$ \\
\hline
\end{tabular}

\section{Simulation procedure}

SOPHE and Xepr of the XSopheSophe-XeprView ${ }^{\circledR}$ computer simulation software suite $(\mathrm{v} .1 .1 .3)^{1)}$ were utilized in this present work. SOPHE is a data entry for generating and running an EPR spectral input file. Xepr is the Bruker spectrometer's data manipulation or acquisition software. This program also controls the spectrometer before working on the data. The output of SOPHE was then loaded into Xepr for further work. Thus, data for simulation can be loaded into Xepr either directly as obtained from the spectrometer or generated from the data entry file in SOPHE. In this work, an input data was generated in SOPHE using the command line and the output was then directly loaded into Xepr for further work.

\section{Work in SOPHE}

In this work, $\ell$ - $\alpha$-alanine simulated spectra due to the resonance of SAR, R2 and two conformations of R3 radical were fitted to X-band CW-EPR resonance spectra of the first-derivative of the absorption lines of $28 \mathrm{kGray}, \gamma$-irradiated of homegrown $\ell$ - $\alpha$-alanine single crystal that were recorded at RT. To do that, using data provided in Tables 1-3, the author must generate SOPHE input files of all radicals, each due to four sites per unit cell. For example, to simulate an experimental spectrum when the magnetic B field was parallel to a crystal axis, it requires 4 (obtained from 4x1) different SOPHE data files, and therefore 4 simulated spectra, for proceeding further. The " 4 " number in the bracket was of the SAR, R2 and two conformations of R3's contributions, whereas "1" means that the 4-files simply have the same resonance pattern. It was because all the sites were magnetically equivalent to each other. This spectral simplification was due to application of two-fold screw axes symmetry as described above. Likewise, it requires 8 (obtained from 4x2) different SOPHE files to simulate an experimental spectrum when the B field was on a crystal plane. Thus, it is clear that in order to simulate a CW-EPR $\ell$ - $\alpha$-alanine crystal spectrum, the more arbitrary orientation of an external B field was set with respect to a crystal frame, the more number of simulated files involved, and hence the process more complex. In this work, six different $\ell$ - $\alpha$-alanine crystal experimental EPR spectra (the ones on the Fig.3-4) were simulated to confirm the simultaneous presence of radicals in the system.

SOPHE data files due to SAR were generated from three different nuclei, namely an $\mathrm{H}_{\alpha}$ proton, three-equivalent methyl $\left(\mathrm{H}_{\beta}\right)$ protons and a dipolar coupled hydrogen $\left(\mathrm{H}_{\mathrm{dip}}\right)$ proton (see Table-1 for the nuclei). Thus the spin Hamiltonian of the $\mathrm{SAR}$ is,

$\mathrm{H}_{\mathrm{SAR}}=\beta \mathbf{S} \cdot \mathbf{g} \cdot \mathbf{B}+\mathbf{S} \cdot \mathbf{A} \cdot \mathbf{I}\left[\mathrm{H}_{\alpha}\right]+\gamma \mathbf{B} \cdot \mathbf{I}\left[\mathrm{H}_{\alpha}\right]+\mathbf{S} \cdot \mathbf{A} \cdot \mathbf{I}$

$\left[\mathrm{H}_{\beta}\right]+\gamma \mathbf{B} \cdot \mathbf{I}\left[\mathrm{H}_{\beta}\right]+\mathbf{S} \cdot \mathbf{A} \cdot \mathbf{I}\left[\mathrm{H}_{\text {dip }}\right]+\gamma \mathbf{B} \cdot \mathbf{I}\left[\mathrm{H}_{\text {dip }}\right]$

Here $\mathbf{S}$ and $\mathbf{I}$ are the electronic and nuclear spins, respectively. $\mathbf{A}$ is a hyperfine coupling tensor, $\beta$ is the Bohr magneton and $\gamma$ is proton gyromagnetic ratio.

Whereas SOPHE files due to R2 radical were developed from four protons, namely three-equivalent methyl $\left(\mathrm{H}_{\beta}\right)$ protons and three amino $\mathrm{H}_{\beta(\mathrm{N})}$ protons (see 
Table-2 for the nuclei). The spin Hamiltonian due to this radical is, $\mathrm{H}_{\mathrm{R} 2}=\beta \mathbf{S} \cdot \mathbf{g} \cdot \mathbf{B}+\mathbf{S} \cdot \mathbf{A} \cdot \mathbf{I}\left[\mathrm{H}_{\beta}\right]+\gamma \mathbf{B} \cdot \mathbf{I}\left[\mathrm{H}_{\beta}\right]+\mathbf{S} \cdot \mathbf{A} \cdot \mathbf{I}\left[\mathrm{H}_{\beta 1}\right]$ $+$

$\gamma \mathbf{B} \cdot \mathbf{I}\left[\mathrm{H}_{\beta 1}\right]+\mathbf{S} \cdot \mathbf{A} \cdot \mathbf{I}\left[\mathrm{H}_{\beta 2}\right]+\gamma \mathbf{B} \cdot \mathbf{I}\left[\mathrm{H}_{\beta 2}\right]+\mathbf{S} \cdot \mathbf{A} \cdot \mathbf{I}\left[\mathrm{H}_{\beta 3}\right]+$ $\gamma \mathbf{B} \cdot \mathbf{I}\left[\mathrm{H}_{\beta 3}\right]$

SOPHE files due to two conformations of $\mathrm{R} 3$ radical; each was developed from a methyl $\left(\mathrm{H}_{\beta}\right)$ proton (see Table-3 for the proton). The spin Hamiltonian is (meaning of the symbols are the same as before),

$\mathrm{H}_{\mathrm{R} 3}=\boldsymbol{\beta} \mathbf{S} \cdot \mathbf{g} \cdot \mathbf{B}+\mathbf{S} \cdot \mathbf{A} \cdot \mathbf{I}\left[\mathrm{H}_{\beta}\right]+\gamma \mathbf{B} \cdot \mathbf{I}\left[\mathrm{H}_{\beta}\right]$

To enable exact comparison between experimental and simulated spectrum, some instrumental parameters such as external magnetic field center and microwave field orientations $(\theta$ and $\phi$ angles) to the crystal frame were accounted for in the calculation of the simulated spectrum. At the completion of running a SOPHE file, the SOPHE program scaled the output spectrum (stored as .sph file) to fit the integer range $\left( \pm 1 \times 10^{+4}\right)$ and saved the factor as the receiver gain (RRG) in a .par file. The RRG's data was then extracted manually for further work (spectral addition). This spectral addition facility is provided in order to accommodate any mixtures of paramagnetic sites in a system, as found in $\ell$ - $\alpha$-alanine system.

\section{Work in Xepr}

Spectral normalization and addition were carried out in Xepr by making use of an RRG's data extracted from a SOPHE .par file. A SOPHE output spectrum was loaded into Xepr before spectral intensities were divided by an RRG data for normalization purpose. The normalized $\mathrm{R}_{1}$ spectrum is $\mathrm{R} 1 / \mathrm{RRG}(1)=\mathrm{xR}_{1}$. Two spectra were added with the one in the primary and the other in the secondary view ports. The addition result of two normalized spectra $\left\{\mathrm{xR}_{1}\right\}$ and $\left\{(1-\mathrm{x}) \mathrm{R}_{2}\right\}$ is $\left\{x R_{1}+(1-x) R_{2}\right\}$. As discussed, along a crystal axis all the four sites exhibit the same spectrum $\mathrm{R}$, thus the normalized $\mathrm{R}$ spectrum is $x R$. Further, since the spectrum of each radical in the system may not be created with the same efficiency and also not contain the same number of spins, determination of correct proportionality is very important in spectral addition. In this work, two possible proportions of SAR, R2 and two conformations R3 radical were tried, that is 55\%: $35 \%: 5 \%: 5 \%$ and $50 \%$ : 30\%: 10\%: 10\%. By loading the normalized SAR and R2 spectral files, each in the secondary and primary view ports, gave $\mathrm{R}_{(\mathrm{SAR}+\mathrm{r} 2)}$ ' (Eq.5), followed by the (SAR+R2)' and R3' files, gave $\mathrm{R}_{\text {total }}$ (Eq.6). $R_{\text {total }}$ in Eq. (6) is the output of Xepr and therefore the simulation outcome. Thus the $\mathrm{R}_{\text {total }}$ is now ready to compare to the associated experimental spectrum.

$$
\begin{aligned}
& \mathrm{R}_{(\mathrm{SAR}+\mathrm{R} 2)^{\prime}}=0.5 \mathrm{R}_{\mathrm{SAR}^{\prime}}+0.3 \mathrm{R}_{\mathrm{R} 2}, \\
& \mathrm{R}_{\text {total }}=0.8 \mathrm{R}_{(\mathrm{SAR}+\mathrm{R} 2)^{\prime}}+0.2 \mathrm{R}_{\mathrm{R} 3},
\end{aligned}
$$

\section{Simulation results and discussion}

The external B field // to crystal axes

Figures 5-7 show step-by-step addition of the SAR, R2 and R3 spectra when the B field was parallel to $\langle a>$ axis. Figures $8 \mathrm{a}-\mathrm{b}$ give similar pictures, but shortened stages when the B field was parallel to $\langle b\rangle$ axis, while Figures $9 a-b$ depict the spectra when the $\mathrm{B}$ field was parallel to $\langle\mathrm{c}\rangle$ axis. Throughout the work, spectra from site-1 were occupied. For each radical, the linewidth parameter was set such that the simulated spectrum approximates to the experimental spectra. The distribution ratio of 0.5: 0.3: 0.2 was used to add the spectra of SAR, R2 and two conformations of R3 radical.

In Fig. 5a, (011R1+u) and (0017R2+u) were, respectively, the normalized SAR and R2 spectra, with the 1/RRG values of 0.11 and $0.017 ;+\mathrm{u}$ indicating the uncertainty of the direction cosines of the tensors in the calculation. Those spectra were added together with the proportion, each of 0.5 and 0.3 . The result is "total(05R1:03R2)+u" spectrum (see Fig. $5 \mathrm{~b})$. Each of the resonance line in Fig. $5 \mathrm{~b}$ 
has been marked with a number, indicating to which radical it was assigned. For example, "1" means a resonance line due to SAR, while "2" was due to R2 radical; a superscript + or - indicating whether the line was assigned to the $M_{s}=+1 / 2$ or $-1 / 2$ of the electronic energy states belong to the system. 5a)

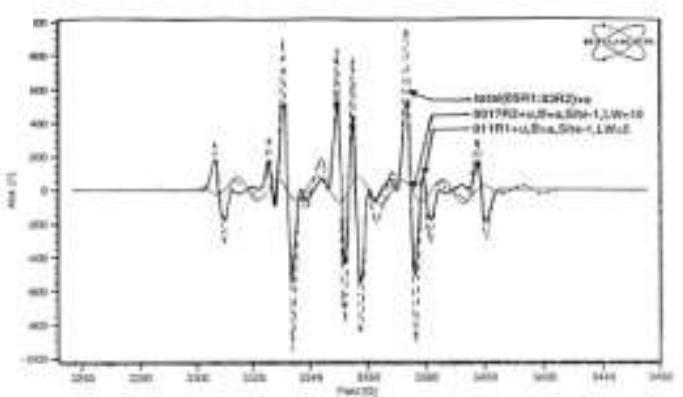

5b)

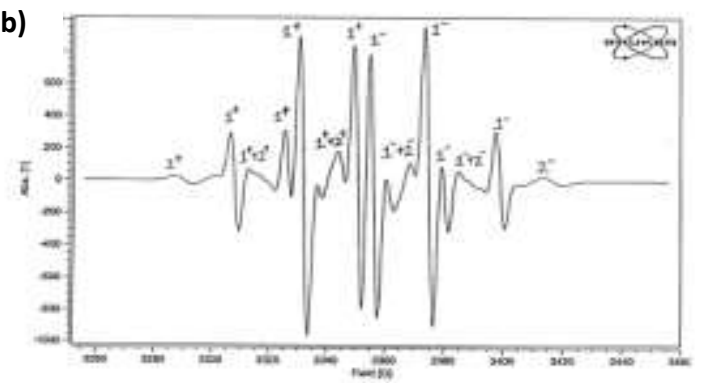

Fig. 5: a) Addition between the normalized site- 1 spectrum of the SAR: $(011 R 1+u)$ and that of R2 radical: $(0017 R 2+u)$ when the external $B$ field was parallel to $<a>$ axis. The $L W_{S A R}=5 \mathrm{MHz}$ and $L_{\mathrm{R} 2}=10 \mathrm{MHz}$. The result (the dash spectrum) was 'total(05R1:03R2)+u'. b) The 'total(05R1:03R2)+u' spectrum reprinted from the dash spectrum in a). The resonance lines have been marked as described in the text above.

Figure 6 illustrates spectral addition between two conformations of R3 radical (R3 and R3prime). Spectral parameters and conditions occupied during the simulation process were also labeled. The addition result (the dash line) is 'total(R3+R3prime)' spectrum. Fig. 7a shows how to add the 'total(05R1:03R2)+u' spectrum given in Fig. $5 b$ and the 'total(R3+R3prime)' spectrum in Fig. 6, and the results is provided in Fig. 7b. It is clear from Fig. 7a
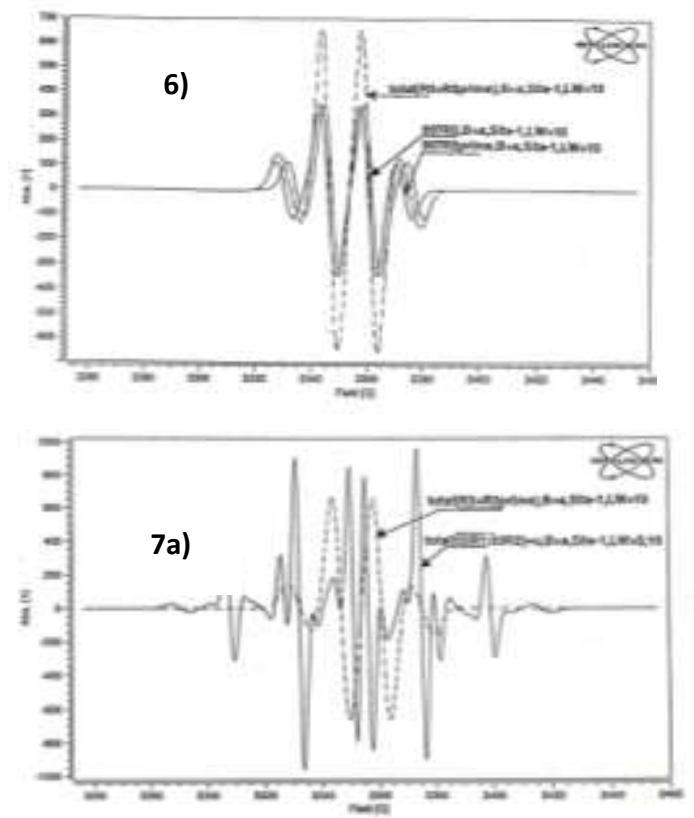

that the minority radical species $\mathrm{R} 3$ and R3prime contribute mainly to the central resonance lines. The spectrum shown in Fig. 7b is therefore the outcome of spectral simulation when the B field was parallel to $<$ a $>$ crystal axis. The other outcomes are performed in Fig. $8 \mathrm{~b}$ and $9 \mathrm{~b}$. Note that the spectra in Fig. 14b-16b are then called as "axial" spectra; refer to the simulated spectra when the external B field was parallel to crystal axes.

g. 6: The site-1 spectral addition between two informations R3 (R3 and R3prime). The result (dash ie) was 'total(R3+R3prime)' spectrum. The B field as parallel to $<a>$, the $\mathrm{LW}=10 \mathrm{MHz}$.

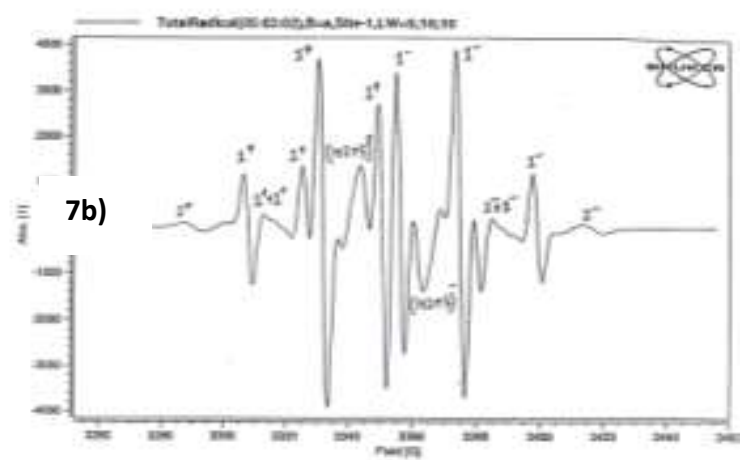



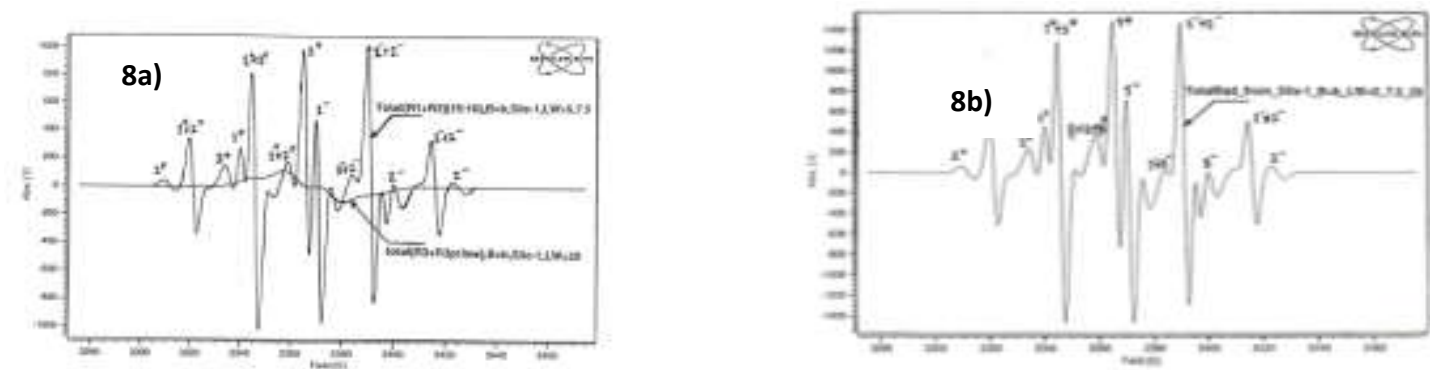

Fig. 8: a) Spectral addition between (SAR and $R 2$ ) 'total(R3+R3prime)' spectrum when the external B field $n$ marked as described in the text. Note that the $\mathrm{LW}_{\mathrm{SAR}}=5 \mathrm{MHz}$ and $\mathrm{LW}_{\mathrm{R} 3}=20 \mathrm{MHz}$. b) The outcome of a). This outcome was compared with the $(B / /<b>)$ experimental spectrum. See Fig. 10b for the comparison.
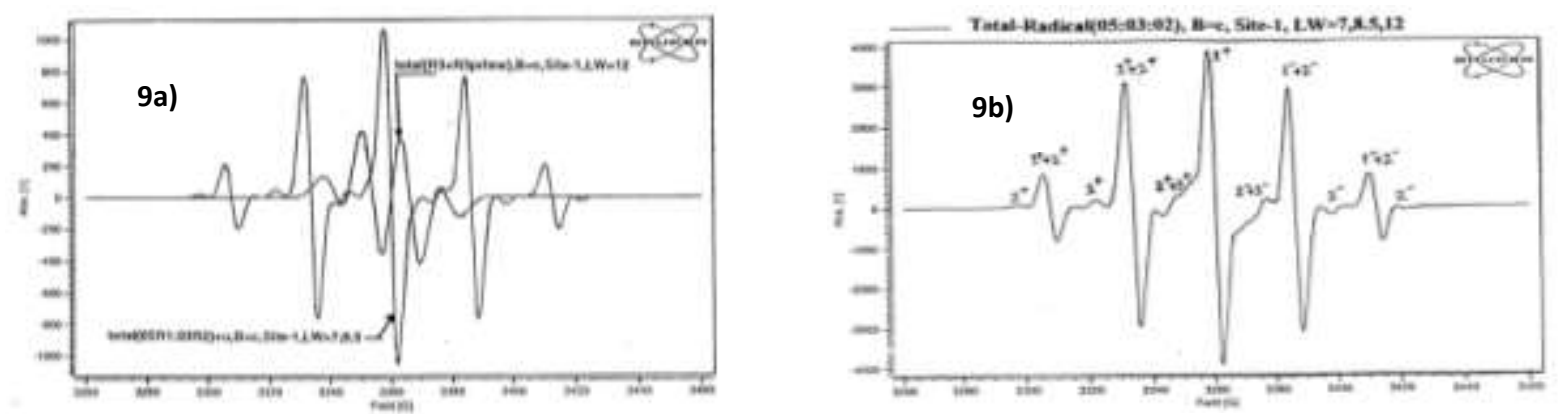

Fig. 9: a) As before, spectral addition due to $S A R, R 2$ and two conformations of R3 radicals, but when the $B / /\langle C\rangle$ axis. Simulation parameters are labeled on the spectra. b) The spectral outcome of a). See Fig. 10c for comparison with the associated experimental spectrum.

Figures 10a-c below show the comparison between the outcome of simulation and the associate experimental spectra. As shown, all the spectral features come out on the simulated spectra, indicating that the parameters occupied during the simulation, and hence the proposed radical structures, can reproduce the composite experimental spectra. Further, the existence of the R3 radical, in

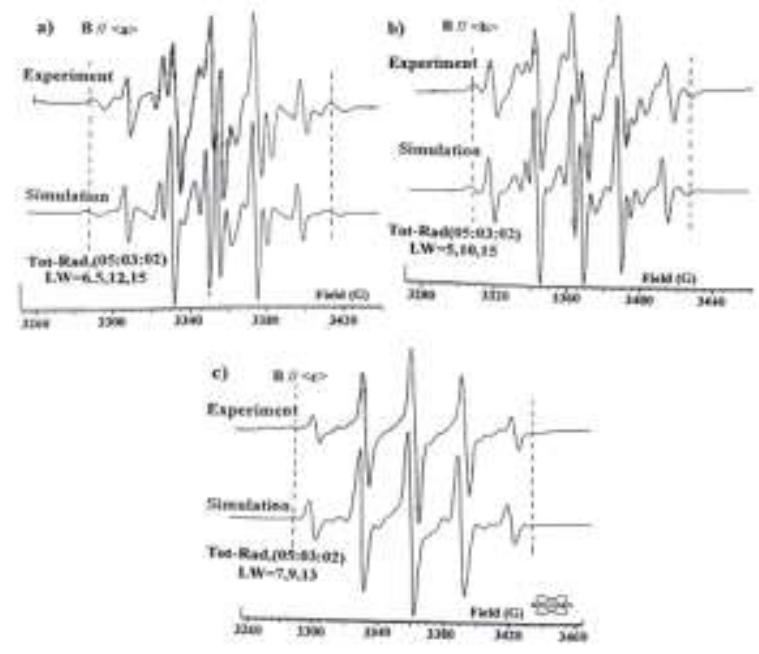

minor proportion, was verified in the simulation outcome and the comparable amounts of the main radicals, SAR and R2 radical, were also apparent. Therefore, as a general conclusion, it can be said that exact proportion of the constituent radicals is required to obtain a correct simulated $\mathrm{CW}$ EPR/ $\ell-\alpha$-alanine single crystal spectra.

Fig. 10: Comparison between first-derivative $X$-band CW-EPR $/ \gamma$-irradiated at room temperature of $\ell$ - $\alpha$-alanine single crystal experimental and simulated spectra, when: a) B // $\langle$ a $>$, b) B $/ /\langle$ b $>$ and c) B $/ /<$ c $>$ axis. Parameters occupied during simulations were as in the text. The LW setting and the relative proportion of the constituent radicals were labeled on each spectrum, where for example 6.5, 12, 15 mean that the LW's settting of the SAR, R2 and R3 radicals were $6.5 \mathrm{Mhz}, 12 \mathrm{MHz}$ and $15 \mathrm{MHz}$, respectively. 
The external B field on crystal planes

Figure 11 depicts the simulated spectra as in Fig. 10, but the external B field was set on $a-b, b-c$ and $c-a$ crystal planes, thereafter these spectra were called as "planar" spectra. For each constituent radical, spectral addition in Fig. 11 was carried out from two different sites whose resonance patterns were not equivalent of each other, namely (site-1+site-3) for B field on $a-b$ plane, (site-1+site-2) both for $\mathrm{B}$ field on $\mathrm{b}-\mathrm{c}$ and $\mathrm{c}-\mathrm{a}$ planes. The relative amount of 0.5: 0.3: 0.2 for SAR, R2 and two conformations of $\mathrm{R} 3$ radical was also followed in this calculation. As shown, however, the planar spectra did not fit exactly to the experimental spectra. Further, using given radical's linewidth, the main (five) resonance lines of all three planar spectra were simulated and it was agree with the experimental spectra. Unfortunately, in some results, the "satellites" accompanied each of the main lines have different intensities, or even overlap with each other. These discrepancies might be attributed to imprecise setting of the spectral linewidths. The linewidths may depend on theta $(\theta)$ and phi $(\phi)$ values of the external magnetic field and also on the nuclear $\left(\mathrm{M}_{\mathrm{I}}\right)$ energy state values. As mentioned before, in this work, the linewidth parameters were estimate only and the settings used were the closest values, and hence the best results to the experiment.
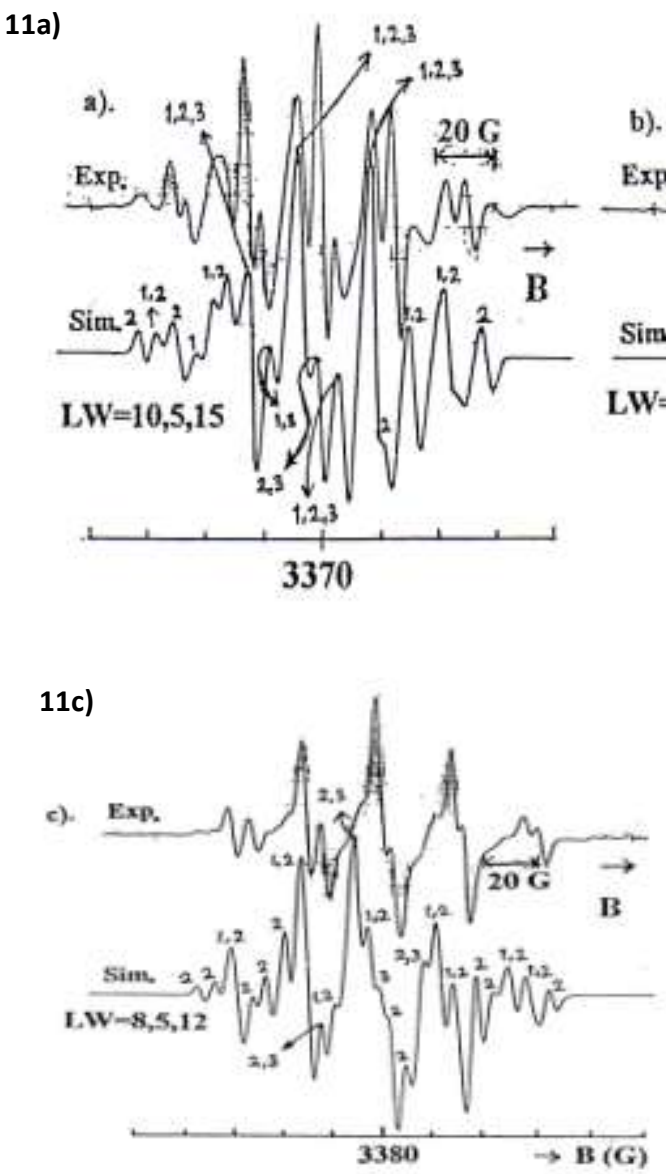

11b)

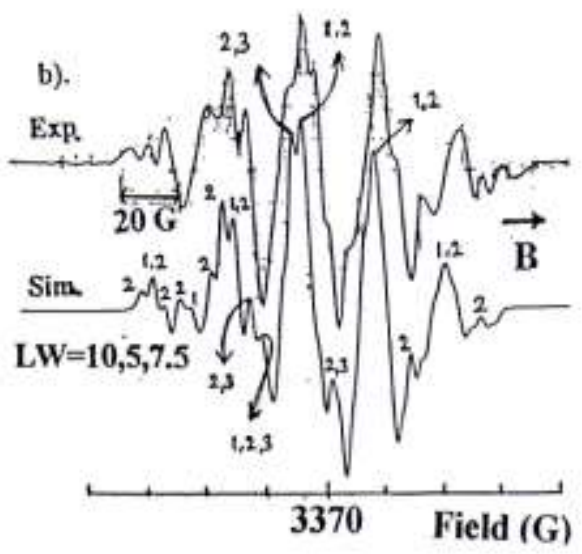

zure-11: Similar comparison as in Fig. 10 it the external $B$ field was on above: a) ab) b-c, and left: c) c-a crystal plane, spectively. The resonance lines due to ch constituent radical were labeled to ow exact comparison with the perimental spectra.
In addition, in order to anticipate any possibility of mis-orientation of the crystal in the cavity during the experiment and hence to obtain a correct simulation result, some efforts had been made previously by the author. This includes: (i) re-checking the spectral saturation due to SAR and R2 radical; (ii) Checking that the crystal 
orientation for SAR corresponds to a maximum hyperfine splitting; (iii) Rotating the crystal both in clockwise and anticlockwise directions within the uncertainty of the goniometer scale; (iv) Varying the spectral linewidth of the individual radicals. Result of these efforts were presented and discussed in the following paragraphs.

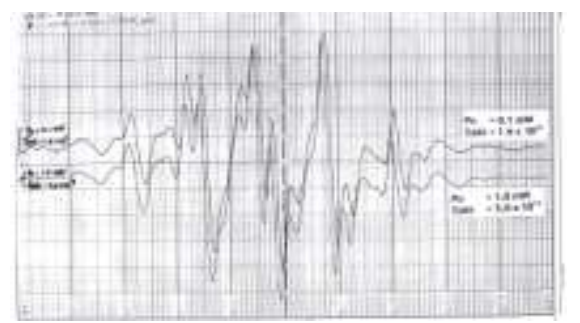

(ii) The maximum hyperfine splitting

In the $\ell$ - $\alpha$-alanine system, the principal values of $\mathrm{H}_{\alpha}$ tensor of the SAR gave rise to the maximum hyperfine splitting. Thus the total spectral span along this particular crystal orientation is the maximum, if it was assumed that the values of the $\mathrm{H}_{\beta}$ tensors were almost isotropic (refers back to the matrices of the transformed $\mathrm{H}_{\alpha}$ and $\mathrm{H}_{\beta}$ tensors in Table-1). Therefore, in this work the goniometer was adjusted until the

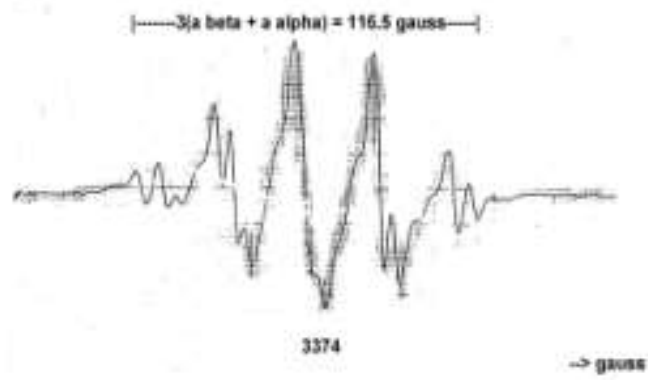

(iii) Clockwise and anti-clockwise rotation of crystal

To make correct measurements on the goniometer, and hence, to record a correct spectrum, the crystal was rotated twice, (i) Spectral saturation

Based on the experimental results depicted in Fig. 12, it was clear that SAR and R2 spectra did not saturate at the microwave power setting of $1 \mathrm{~mW}$. As shown, lowering the microwave power by a factor 10 to $0.1 \mathrm{~mW}$ and increasing the gain by 3 gave rise to unchanged spectral height, which means that the experimental spectrum occupied in the work was not saturated

yet.

Fig. 12: Spectral saturation. $\ell-\alpha$-Alanine experimental spectra due to SAR and R2 radical measured at room temperature and the $B$ field $/ /\langle a\rangle$. The protocol was: $3380 / 200$ gauss, 0.4 gauss, $4.0 \mathrm{sec}$ and 16 $\mathrm{min}$. The microwave power and the gain setting were: a) $0.1 \mathrm{~mW}$ and $1.6 \times 10^{+5}$, b) $1.0 \mathrm{~mW}$ and $5 \times 10^{+4}$.

SAR lines reach the maximum splitting by working out the angles from the direction cosines of the largest hyperfine. Further, selecting the spectrum with the greatest span due to lone site was carried out. The aim was to accommodate the existence of the four non-equivalent sites that may not have splitting maximized together. Fig. 13 shows the spectrum with the greatest span of the hyperfine splitting due to $\mathrm{H}_{\alpha}$ tensor of the SAR, 116.5 gauss.

Fig. 13: The maximum hyperfine splitting. $\mathrm{l}-\alpha-$ Alanine experimental spectrum due to $H_{\alpha}$ tensor of the SAR with the greatest span of $\left(3 \mathrm{a}_{\beta}+\mathrm{a}_{\alpha}\right)=116.5$ gauss, measured at room temperature. The external $B$ field was in $\theta=65.5^{\circ}, \phi=117.0^{\circ}$ on the goniometer scale. The protocol was $3374 / 200$ gauss, $1 \mathrm{~mW}$, $5 \times 10^{+4}, 0.4$ gauss, $4.0 \mathrm{sec}$ and $16 \mathrm{~min}$.

first clockwise and then anti-clockwise, and the result was two similar spectra (see Fig.14), indicating that the spectrum under investigation was indeed the correct spectrum. 


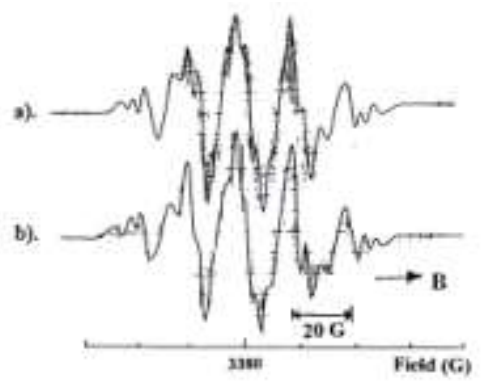

(iv) Spectral linewidth determination

Linewidth of an EPR spectrum due to different radicals are the resultant of individual linewidths. The individual linewdiths are not necessarily identical. As mentioned above, in this work the linewidth parameter was set such that the simulation outcome approximates to the experimental results. Since it was difficult to determine the linewidth of the individual radicals, determination of this parameter was essentially one of trial and error. The value was assumed and generated for the other radicals. As the first trial, it was assumed to be $5 \mathrm{MHz}$ and then the value was varied for the other radicals by factors $1.5,1.6,2,2.5,3$ or even 4 . The most suitable linewidths were occupied in the simulation work and the values were labeled on each spectrum.

\section{Conclusion}

A general comment on the simulation results gathered throughout this work: it was quite difficult to try for a fit to the $\ell-\alpha-$ alanine experimental spectrum when the external B field was on a crystal plane. This difficulty might be attributed to first, the property of crystal symmetry relating paramagnetic sites that seems to be easily removed by a non-expert touch of the goniometer. One effect is to overlap the hyperfine features of the radicals, and therefore, to broaden the resonance of the spectrum. Second, it was essential to check the ENDOR data, since it was the source data for this work.
Fig. 14: Two similar $\ell$ - $\alpha$-alanine experimental spectra measured at room temperature by imposing the external $B$ field on $a-b$ plane: $\theta=55^{\circ}, \phi=90^{\circ}$. The crystal orientation obtained from: a) clockwise and b) anticlockwise rotations of the goniometer. The protocol was 3380/200 gauss, $1 \mathrm{~mW}$, $5.0 \times 10^{+4}, 0.4$ gauss, $4.0 \mathrm{sec}$. and $16 \mathrm{~min}$. The spectrum in Fig. 14a) was the one occupied in Fig. 11b.

\section{Acknowledgment}

The author would like to thank Prof. Grame Hanson of CMR UQ for the SOPHE program.

\section{References}

1) Hanson, G.R.; Gates, K.E.; Noble, C.J.; Griffin, M.; Mitchell, A. and Benson, S. "XSophe-Sophe-XeprView ${ }^{\circledR}$. A computer simulation software suite (v. 1.1.3) for the analysis of continuous wave EPR spectra". Journal of Inorganic Biochemistry. 98, 5, pages 903-916, 2004.

2) Sagstuen, E., Hole, E.O.; Haugedal, S.R.; Nelson, W.H. "Alanine radicals: Structure determination by EPR and ENDOR of single crystals X-irradiated at $295 \mathrm{~K}^{\prime}$. Journal of Physical Chemistry A. 101, 50, pages 9763-9772, 1997.

3) Kuroda, S. and Miyagawa, I. "ENDOR study of an irradiated crystal of $\ell$-alanine: Environment of the stable $\mathrm{CH}_{3} \dot{\mathrm{C}} \mathrm{HCO}_{2}{ }^{-}$ radical". Journal of Chemical Physics. 76, pages 3933-3944, 1982.

4) Pilbrow, JR. et al. "Pulsed EPR investigation of hyperfine structure in $\gamma$ irradiated alanine". Applied Radiation and Isotopes, 47, 11-12, pages 1257-1261, 1996.

5) Sagstuen, E.; Hole, E.O.; Haugedal, S.R.; Lund, A.; Eid, O.I.; Erickson, R. "EPR and ENDOR analysis of $X$-irradiated $\ell$-alanine and $\mathrm{NaHC}_{2} \mathrm{O}_{4} \cdot \mathrm{H}_{2} \mathrm{O}$. Simulation of microwave power dependence of satellite lines". Nucleonika, 42, 2, pages 353-372, 1997.

6) Nye, J.F. "Physical properties of crystals: Their representations by tensors and matrices". Oxford University Press, Amen House, London E.C.4, pages 11-12, 1957. 